\title{
Multiscale probabilistic modeling of a crack bridge in glass fiber reinforced concrete
}

\author{
R. Rypl ${ }^{a}$, M. Vořechovskýa,*

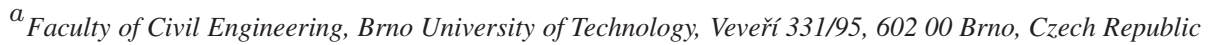

Received 2 December 2016; accepted 4 April 2017

\begin{abstract}
The present paper introduces a probabilistic approach to simulating the crack bridging effects of chopped glass strands in cement-based matrices and compares it to a discrete rigid body spring network model with semi-discrete representation of the chopped strands. The glass strands exhibit random features at various scales, which are taken into account by both models. Fiber strength and interface stress are considered as random variables at the scale of a single fiber bundle while the orientation and position of individual bundles with respect to a crack plane are considered as random variables at the crack bridge scale. At the scale of the whole composite domain, the distribution of fibers and the resulting number of crack-bridging fibers is considered. All the above random effects contribute to the variability of the crack bridge performance and result in size-dependent behavior of a multiply cracked composite.
\end{abstract}

(c) 2017 University of West Bohemia. All rights reserved.

Keywords: glass fiber reinforced concrete, probability, crack bridging, tensile strength

\section{Introduction}

Glass fibers as reinforcement in cement-based matrix were first utilized in the 1960s in Russia [2]. A further major step towards glass fiber reinforced concrete (GFRC or GRC) was the development of alkali-resistant (AR) glass which was achieved by increasing the content ( $>16 \%)$ of zirconia $[9,18]$. This enhancement allowed for the production of a durable high-performance cement-based composite, which has been used in various modifications in structural and military engineering since $[1,13]$.

Each of the AR-glass fibers is essentially a bundle of monofilaments (typically 50 to 400) which are bonded together by a sizing material. When bridging a crack, these filaments debond and rupture or are being pulled out and thus increase the toughness of the cement-based composite [10]. Moreover, the short dispersed fibers increase the first cracking stress and, above a critical volume fraction threshold, the ultimate tensile strength. These features together with the enhanced durability make the use of GFRC an alternative to traditional steel fiber reinforced concrete (FRC). However, the bridging mechanism is far more complex than in FRC.

Once a crack has been formed in the matrix, the glass fibers bridging the crack act against further crack opening by stretching and pullout. During this process, some filaments are completely pulled out while others rupture. The mechanism exhibits random features that can be divided into three scales:

\footnotetext{
*Corresponding author. Tel.: +420 541147 370, e-mail: vorechovsky.m@vut.cz. https://doi.org/10.24132/acm.2017.334
} 
1) At the micro scale, individual filaments within a bundle experience random fiber-matrix interfacial shear stress that transfer the tensile force from the filament to the neighboring matrix. The stress transfer depends on filament position within the bundle and thus on the penetration of the matrix into the bundle core. A second source of randomness at the micro scale is the fiber strength that is determined by the weakest flaw in the material structure and also by variations in the filament cross-section.

2) At the meso scale, individual bridging fibers are randomly oriented and positioned within the composite domain. This randomness causes variability in the bridging force due to snubbing and non-uniform pullout lengths [8].

3) At the macro scale, the overall number of fibers bridging a crack is a random variable that depends on the specimen geometry, fiber geometry, fiber volume fraction and also on production of specimens such as the casting process.

A model that considers these sources of random effects and reflects the complexity and unique bridging mechanism of the short glass fiber bundles does not exist to date.

The objective of this paper is to introduce two approaches to modeling of a crack bridge in GFRC: In Section 2, a probabilistic approach that predicts statistical moments of the bridging force is described. In Section 3, a discrete model with semi-discrete representation of the fiber bundles is presented. Both models are compared in a computational example in Section 4 and their possibilities and limitations are discussed in Section 5.

\section{Probabilistic model}

The semi-analytical probabilistic model is limited to uniaxial tensile loading of a composite with discrete, planar matrix cracks and mechanically independent fibers. The mechanical independence of fibers is provided if matrix deformations are much lower than the fiber deformations i.e. the matrix stiffness is much higher than that of the fibers: $E_{\mathrm{m}}\left(1-V_{\mathrm{f}}\right) \gg E_{\mathrm{f}} V_{\mathrm{f}}$. Here, $E_{\mathrm{m}}$ and $E_{\mathrm{f}}$ are the matrix and fiber elastic moduli, respectively, and $V_{\mathrm{f}}$ is the fiber volume fraction.

The model distinguishes three models for three levels of resolution of the composite: (a) composite crack bridged by bundles of filaments, (b) a single filament bundle and (c) a single filament, see Fig. 1.

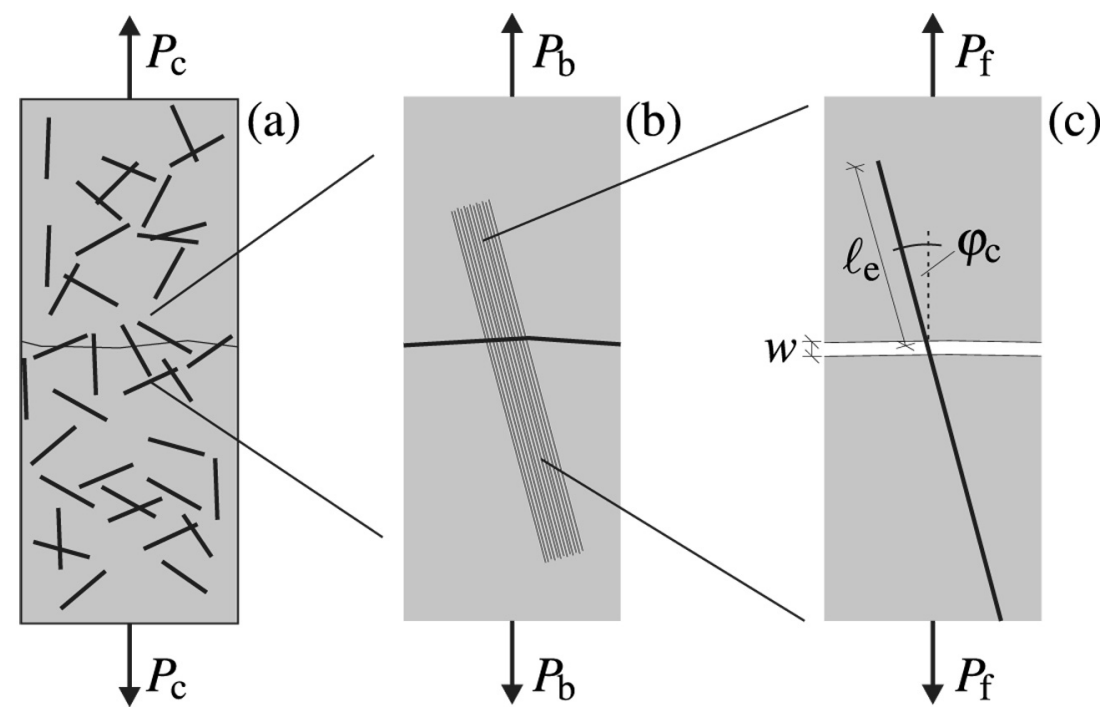

Fig. 1. Multiscale approach to the modeling of GFRC: (a) composite crack bridge with multiple filament bundles; (b) filament bundle; (c) single filament considered independently from the bundle 


\subsection{Single filament}

Let us assume that the bridging action of a single filament with embedded length $\ell_{\mathrm{e}}$ and inclination angle $\varphi_{\mathrm{c}}$ (with respect to the crack plane normal) is provided in the form

$$
P_{\mathrm{f}}=f\left(w, \ell_{\mathrm{e}}, \varphi_{\mathrm{c}}, \boldsymbol{\theta}_{\mathrm{r}}, \boldsymbol{\theta}_{\mathrm{d}}\right),
$$

where $P_{\mathrm{f}}$ is the bridging force, $w$ is the crack opening, $\boldsymbol{\theta}_{\mathrm{d}}$ is a vector of deterministic parameters and $\boldsymbol{\theta}_{\mathrm{r}}$ a vector of random variables defined over the sampling space $\Omega_{\mathrm{r}}$ with the corresponding joint cumulative distribution function (CDF) $G_{\Omega_{\mathrm{r}}}$. The mean force transmitted by a filament within a bundle bridging a matrix crack is

$$
\mu_{P_{\mathrm{f}}}\left(w, \ell_{\mathrm{e}}, \varphi_{\mathrm{c}}\right)=\mathrm{E}_{\Omega_{\mathrm{r}}}\left[P_{\mathrm{f}}\right]
$$

with $\mathrm{E}_{\Omega}[\mathbf{X}]$ being the expectation operator applied to the random variable $\mathbf{X}$ defined over the sampling space $\Omega$ with the joint $\mathrm{CDF} G_{\Omega}(\mathbf{X})$, i.e.,

$$
\mathrm{E}_{\Omega}[\mathbf{X}]=\int_{\Omega} \mathbf{X d} G_{\Omega}(\mathbf{X}) .
$$

The variance of the filament bridging force is given by

$$
\sigma_{P_{\mathrm{f}}}^{2}\left(w, \ell_{\mathrm{e}}, \varphi_{\mathrm{c}}\right)=\mathrm{D}_{\Omega_{\mathrm{r}}}\left[P_{\mathrm{f}}\right]
$$

with $\mathrm{D}_{\Omega}[\mathbf{X}]$ being the variance operator applied to the random variable $\mathbf{X}$ defined over the sampling space $\Omega$ with the joint $\mathrm{CDF} G_{\Omega}(\mathbf{X})$, i.e.,

$$
\mathrm{D}_{\Omega}[\mathbf{X}]=\mathrm{E}_{\Omega}\left[\mathbf{X}^{2}\right]-\mathrm{E}_{\Omega}[\mathbf{X}]^{2}=\int_{\Omega} \mathbf{X}^{2} \mathrm{~d} G_{\Omega}(\mathbf{X})-\mathrm{E}_{\Omega}[\mathbf{X}]^{2}
$$

\subsection{Filament bundle}

Given the number of filaments in a bundle, $N_{\mathrm{f}}$, the force transmitted by the whole bundle reads

$$
P_{\mathrm{b}}=\sum_{i=1}^{N_{\mathrm{f}}} P_{\mathrm{f}}\left(w, \ell_{\mathrm{e}}, \varphi_{\mathrm{c}}, \boldsymbol{\theta}_{\mathrm{r}, i}, \boldsymbol{\theta}_{\mathrm{d}}\right),
$$

where $\theta_{\mathrm{r}, i}$ is the vector of parameters obtained as the $i$ th sample from the sampling space $\Omega_{\mathrm{r}}$ of the random variables $\theta_{\mathrm{r}}$. Since the inclinations and embedded lengths of the bridging bundles are random, the $\varphi_{\mathrm{c}}$ and $\ell_{\mathrm{e}}$ parameters are to be treated as random variables. Their sampling space is referred to as $\Omega_{\varphi}$. The mean bridging force transmitted by a bundle has the form

$$
\mu_{P_{\mathrm{b}}}(w)=\mathrm{E}_{\Omega_{\varphi} \Omega_{\mathrm{r}}}\left[P_{\mathrm{b}}\right]=N_{\mathrm{f}} \mathrm{E}_{\Omega_{\varphi} \Omega_{\mathrm{r}}}\left[P_{\mathrm{f}}\right] .
$$

For the variance of the bundle bridging force, we have to use the law of total variance, which states

$$
\mathrm{D}[Y]=\mathrm{E}[\mathrm{D}(Y \mid X)]+\mathrm{D}[\mathrm{E}(Y \mid X)]
$$

When this law is applied to the present case, $(Y \mid X)$ is substituted by $P_{\mathrm{b}}\left(w, \ell_{\mathrm{e}}, \varphi_{\mathrm{c}}, \boldsymbol{\theta}_{\mathrm{d}} \mid \boldsymbol{\theta}_{\mathrm{r}}\right)$. One can alternatively express the conditional probability by explicitly writing the integration domain for individual statistical operators in the equation. With this notation, the variability of the randomly oriented filament bundle with random embedded length reads:

$$
\begin{aligned}
\sigma_{P_{\mathrm{b}}}^{2}(w) & =\mathrm{E}_{\Omega_{\phi}}\left[\mathrm{D}_{\Omega_{\mathrm{r}}}\left(P_{\mathrm{b}}\right)\right]+\mathrm{D}_{\Omega_{\phi}}\left[\mathrm{E}_{\Omega_{\mathrm{r}}}\left(P_{\mathrm{b}}\right)\right] \\
& =N_{\mathrm{f}}^{2}\left(\mathrm{E}_{\Omega_{\phi}}\left[\mathrm{D}_{\Omega_{\mathrm{r}}}\left(P_{\mathrm{f}}\right)\right]+\mathrm{D}_{\Omega_{\phi}}\left[\mathrm{E}_{\Omega_{\mathrm{r}}}\left(P_{\mathrm{f}}\right)\right]\right),
\end{aligned}
$$

where we do not explicitly write out the dependencies of $P_{\mathrm{f}}$ on its parameters. 


\subsection{Multiple bundles}

Let us now introduce the variable $N_{\mathrm{b}}$, which stands for the number of bundles (chopped strands) bridging a matrix crack. In a composite with randomly dispersed fiber bundles, $N_{\mathrm{b}}$ will be a random variable with sampling space $\Omega_{\mathrm{b}}$. The total force transmitted by all $N_{\mathrm{b}}$ bundles can be written as

$$
P_{\mathrm{c}}=\sum_{j=1}^{N_{\mathrm{b}}} \sum_{i=1}^{N_{\mathrm{f}}} P_{\mathrm{f}}\left(w, \ell_{\mathrm{e}, j}, \varphi_{\mathrm{c}, j}, \boldsymbol{\theta}_{\mathrm{r}, i j}, \boldsymbol{\theta}_{\mathrm{d}}\right)=\sum_{j=1}^{N_{\mathrm{b}}} P_{\mathrm{b}, j},
$$

where $\ell_{\mathrm{e}, j}$ and $\varphi_{\mathrm{c}, j}$ are the $j$ th samples from the $\Omega_{\varphi}$ sampling space, the vector $\theta_{\mathrm{r}, i j}$ is the $i j$ th sample from the sampling space $\Omega_{\mathrm{r}}$ and $P_{\mathrm{b}, j}$ can be expressed as

$$
P_{\mathrm{b}, j}=\sum_{i=1}^{N_{\mathrm{f}}} P_{\mathrm{f}}\left(w, \ell_{\mathrm{e}, j}, \varphi_{\mathrm{c}, j}, \boldsymbol{\theta}_{\mathrm{r}, i j}, \boldsymbol{\theta}_{\mathrm{d}}\right) .
$$

The mean force resulting from the bridging action of randomly dispersed short fiber bundles has the form

$$
\begin{aligned}
\mu_{P_{\mathrm{c}}}(w) & =\mathrm{E}_{\Omega_{\mathrm{b}} \Omega_{\varphi}, \Omega_{\mathrm{r}}}\left[P_{\mathrm{c}}\right]=\mathrm{E}_{\Omega_{\mathrm{b}}}\left[N_{\mathrm{b}}\right] \mu_{P_{\mathrm{b}}}(w) \\
& =\mathrm{E}_{\Omega_{\mathrm{b}}}\left[N_{\mathrm{b}}\right] N_{\mathrm{f}} \mathrm{E}_{\Omega_{\varphi} \Omega_{\mathrm{r}}}\left[P_{\mathrm{f}}\right] .
\end{aligned}
$$

Applying the law of total variance according to (8) with $P_{\mathrm{c}}\left(w, \ell_{\mathrm{e}}, \varphi_{\mathrm{c}}, \boldsymbol{\theta}_{\mathrm{r}}, \boldsymbol{\theta}_{\mathrm{d}} \mid N_{\mathrm{b}}\right)$ substituted for $(Y \mid X)$, the variance of the crack bridging force $P_{\mathrm{c}}$ is obtained as

$$
\begin{aligned}
\sigma_{P_{\mathrm{c}}}^{2}(w) & =\mathrm{D}_{\Omega_{\mathrm{b}} \Omega_{\varphi} \Omega_{\mathrm{r}}}\left[P_{\mathrm{c}}\right]=\mathrm{D}_{\Omega_{\mathrm{b}} \Omega_{\varphi} \Omega_{\mathrm{r}}}\left[\sum_{j=1}^{N_{\mathrm{b}}} P_{\mathrm{b}, j}\right] \\
& =\mathrm{E}_{\Omega_{\mathrm{b}}}\left[\mathrm{D}_{\Omega_{\varphi} \Omega_{\mathrm{r}}}\left(\sum_{j=1}^{N_{\mathrm{b}}} P_{\mathrm{b}, j} \mid N_{\mathrm{b}}\right)\right]+\mathrm{D}_{\Omega_{\mathrm{b}}}\left[\mathrm{E}_{\Omega_{\varphi} \Omega_{\mathrm{r}}}\left(\sum_{j=1}^{N_{\mathrm{b}}} P_{\mathrm{b}, j} \mid N_{\mathrm{b}}\right)\right] .
\end{aligned}
$$

Exploiting the independence of $P_{\mathrm{b}}$ and $N_{\mathrm{b}}$, equation (13) can be simplified to

$$
\begin{aligned}
\sigma_{P_{\mathrm{c}}}^{2}(w) & =\mathrm{E}_{\Omega_{\mathrm{b}}}\left[N_{\mathrm{b}} \cdot \mathrm{D}_{\Omega_{\varphi} \Omega_{\mathrm{r}}}\left(P_{\mathrm{b}}\right)\right]+\mathrm{D}_{\Omega_{\mathrm{b}}}\left[N_{\mathrm{b}} \cdot \mathrm{E}_{\Omega_{\varphi} \Omega_{\mathrm{r}}}\left(P_{\mathrm{b}}\right)\right] \\
& =\mathrm{E}_{\Omega_{\mathrm{b}}}\left[N_{\mathrm{b}}\right] \cdot \mathrm{D}_{\Omega_{\varphi} \Omega_{\mathrm{r}}}\left[P_{\mathrm{b}}\right]+\mathrm{D}_{\Omega_{\mathrm{b}}}\left[N_{\mathrm{b}}\right] \cdot\left(\mathrm{E}_{\Omega_{\varphi} \Omega_{\mathrm{r}}}\left[P_{\mathrm{b}}\right]\right)^{2} .
\end{aligned}
$$

In order to evaluate the statistical moments of the bridging response, the distribution functions of the random variables need to be known. The derivation of distribution functions for individual random variables is out of the scope of the present publication. Let us just note that the fiberin-composite strength distribution is based on the assumption of a compound Pisson-Weibull process which governs the distribution of flaws in the fiber material. The strength distribution is of the Weibull form and depends on fiber geometry and bond law. Readers interested in a detailed derivation are referred to $[12,14]$.

With no further information, e.g. from SEM, the bond strength distribution has to be chosen ad hoc since it depends on a number of uncontrollable factors. In [14], the authors have achieved a fair fit for the bond strength in textile reinforced concrete with Weibull distribution.

The distribution of the number of dispersed short fibers bridging a planar matrix crack is in detail dealt with in $[16,17]$ and has been shown to follow the binomial distribution (or its Poisson limit for a large number of fibers). 


\section{Discrete model}

The use of the discrete model presented in this section serves for a mutual verification of the two model approaches. In this rigid body spring network (RBSN) model the fiber and matrix phase models are both based on a lattice model. The matrix phase is represented by a set of randomly distributed nodes which are interconnected by springs and kinematic constraints. This nodal set for the matrix phase has lattice topology and material properties by the Delaunay/Voronoi tessellations which enable the discretized matrix phase to behave in an elastically homogeneous fashion (Fig. 2a). As shown in Fig. 2b, the matrix element is defined according to the rigidbody-spring concept [4]. The linear and rotational zero-size springs are formed at the centroid $C$ of the area $A_{i j}$ of the Voronoi facet common to nodes $i$ and $j$. The spring set is constrained to nodes $i$ and $j$ via rigid arm constraints.
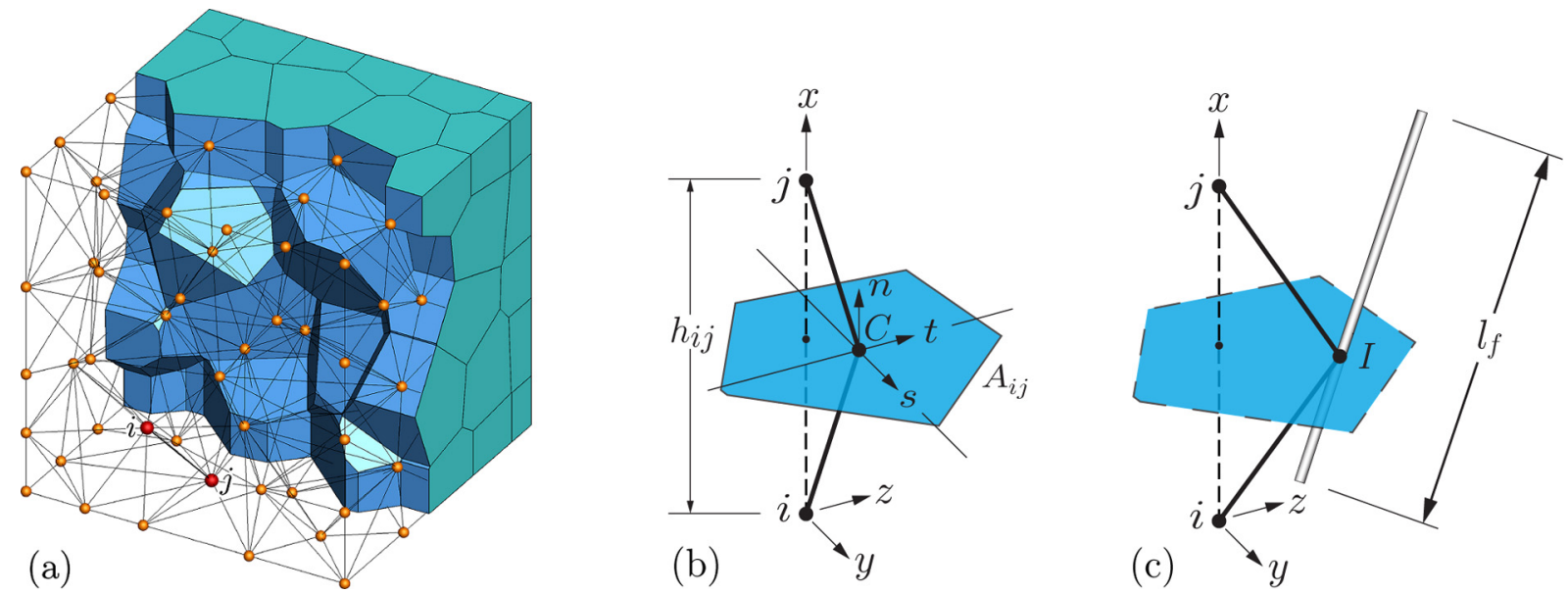

Fig. 2. Lattice discretization of fiber reinforced concrete: (a) Delaunay/Voronoi tessellations of material domain; (b) matrix element $i j$ defined by facet centroid $C$; and (c) fiber element associated with intersection point $I$

A fully-discrete approach considers fibers individually and handles them by addition of degrees of freedom (Fig. 3a). It is suitable only for low number of fibers as the number of degrees of freedom grow linearly with the number of fibers and can thus be used for detailed analyses of laboratory-scale specimens [19].

The most recent semi-discrete approach treats fibers individually but incorporates them into the degrees of freedom of the matrix. The constitutive law of matrix bonds is enhanced by terms accounting for relevant fibers $[6,15]$. Since the fibers do not possess additional degrees of freedom, the semi-discrete approach can be used for problems with relatively high number of fibers [3]. Its standard version called lumped-force includes the fiber mechanical effects directly into cells at the crack faces (Fig. 3b). Such an approach works well for the case of single cracking and coarse discretizations.

When multiple cracking or fine discretizations of the region of interest are present, however, such lumping of the fiber forces is problematic. Kang et al. [5] distribute the transfer of force from fiber to matrix along the embedded lengths of a fiber. This distributed force approach not only solves the aforementioned problems, it also better reflects reality. The distributed forces along the embedded lengths of the fiber are obtained according to the micromechanics of single fiber pullout [11]. The distributed-force model [5] is more realistic as it distributes the force along the fiber path, see Fig. 3c. Because of the more precise representation of the fiber bond, the distributed-force has been used. 
a) fully-discrete

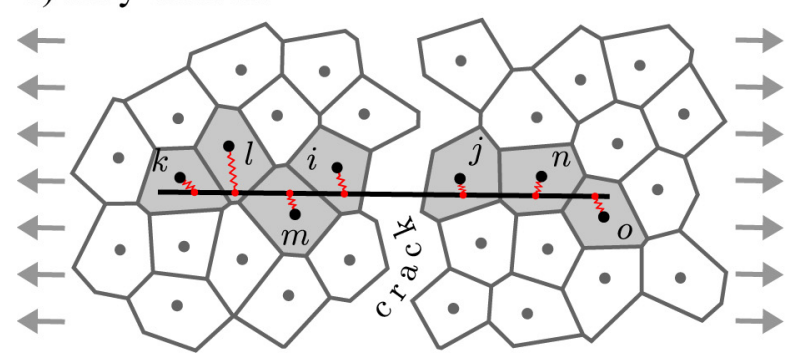

c) semi-discrete distributed-force

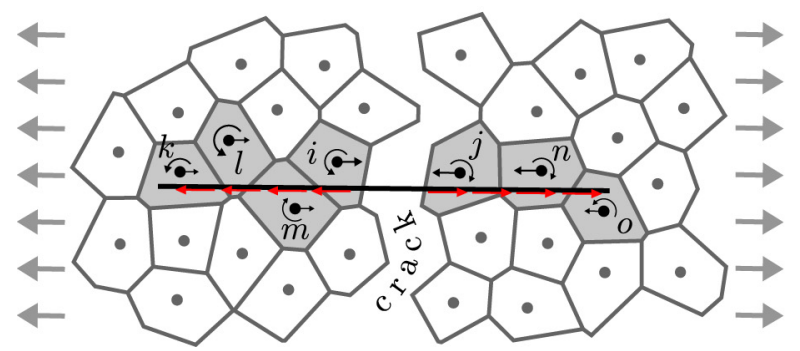

b) semi-discrete lumped-force

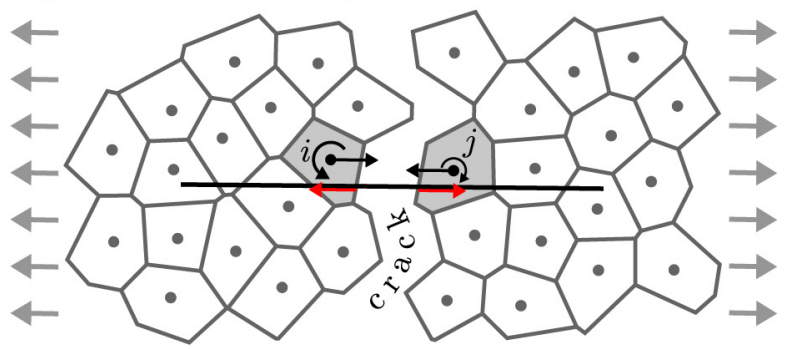

Fig. 3. Various approaches capable to account for contribution of individual fibers to the model response

The fiber phase can be discretized within the computational domain irrespective of the background lattice representing the matrix [5]. A fiber element is defined wherever a fiber passes through the Voronoi facet $A_{i j}$ associated with a matrix element (Fig. 2c). In the semidiscrete fiber model, a linear zero-size spring for the fiber reinforcement is positioned at the intersection point I and aligned with the fiber path. The spring is linked to the associated two nodes $i$ and $j$ through rigid-arm constraints similar to the rigid-body-spring construction of the matrix elements. The semi-discrete modeling of fibers is computationally efficient, contrary to the fully-discrete fiber modeling in which a fiber is discretized as a series of the frame elements with additional nodal degrees of freedom and its elements are linked to the associated nodes via an ordinary bond link. This feature of the semi-discrete fiber model enables simulations with large numbers of fibers.

\section{Computational example}

Having formulated the modeling framework for GFRC in two alternatives, we can proceed to a computational example, which compares the two approaches. Both models require an independent model of a fiber bridging action. For this purpose, we apply the analytical form due to [11] with snubbing and spalling effects according to [7]. For reasons of brevity and readability, we simplify the general expressions by assuming a perfectly plastic (frictional) bond with infinite initial stiffness and constant bond strength. With these assumptions, the resulting form for a filament bridging action in the debonding phase reads

$$
P_{\mathrm{f}, \mathrm{deb}}\left(w, \sigma_{\mathrm{u}}=\infty\right)=A_{\mathrm{f}} \sqrt{\frac{2 E_{\mathrm{f}} \tau w}{r_{\mathrm{f}}}} \cdot \exp \left(f \varphi_{\mathrm{c}}\right) \cdot\left(\cos \varphi_{\mathrm{c}}\right)^{s}
$$

with $E_{\mathrm{f}}, A_{\mathrm{f}}$ and $r_{\mathrm{f}}$ being the filament modulus of elasticity, cross-sectional area and radius, respectively, $\tau$ denoting the bond strength, $f$ the snubbing coefficient and $s$ the spalling coefficient. When the fiber is fully debonded along the embedded length $\ell_{\mathrm{e}}$, the pullout stage starts. Again, for reasons of brevity, we ignore any hardening or softening during the pullout stage and 
write the bridging force during the pullout stage simply as

$$
P_{\mathrm{f}, \operatorname{pull}}\left(w, \sigma_{\mathrm{u}}=\infty\right)=2 \pi r_{\mathrm{f}} \tau\left(\ell_{\mathrm{e}}+w_{0}-w\right) \cdot \exp \left(f \varphi_{\mathrm{c}}\right) \cdot\left(\cos \varphi_{\mathrm{c}}\right)^{s}
$$

with $w_{0}$ being the crack opening at the transition between the debonding and pullout stage. It can be obtained by formulating the continuity condition

$$
P_{\mathrm{f}, \mathrm{deb}}\left(w_{0}\right)=P_{\mathrm{f}, \mathrm{pull}}\left(w_{0}\right) \rightarrow w_{0}=\frac{2 \ell_{\mathrm{e}}^{2} \tau}{r_{\mathrm{f}} E_{\mathrm{f}}} .
$$

In both, equations (15) and (16), the assumption was that fibers have an infinite strength $\sigma_{\mathrm{u}}=\infty$. If we now include the possibility of fiber rupture, we have to multiply the fiber force in the debonding phase by $H\left(\sigma_{\mathrm{u}}-\sigma_{\mathrm{f}}\right)$, where $\sigma_{\mathrm{f}}$ denotes the fiber stress and $H(\cdot)$ the Heaviside step function defined as

$$
H(x)=\left\{\begin{array}{l}
0: x<0 \\
1: x \geq 0
\end{array}\right.
$$

The filament force in the debonding stage then becomes

$$
P_{\mathrm{f}, \mathrm{deb}}(w)=A_{\mathrm{f}} \sqrt{\frac{2 E_{\mathrm{f}} \tau w}{r_{\mathrm{f}}}} \cdot \exp \left(f \varphi_{\mathrm{c}}\right) \cdot\left(\cos \varphi_{\mathrm{c}}\right)^{s} \cdot H\left(\sigma_{\mathrm{u}}-\sigma_{\mathrm{f}}\right)
$$

with

$$
\sigma_{\mathrm{f}}=\frac{P_{\mathrm{f}, \mathrm{deb}}\left(w, \sigma_{\mathrm{u}}=\infty\right)}{A_{\mathrm{f}}} .
$$

In a similar manner, the pullout force has to be multiplied by a Heaviside function which ensures that fibers have not ruptured at their peak stress during the debonding so that

$$
P_{\mathrm{f}, \operatorname{pull}}(w)=2 \pi r_{\mathrm{f}} \tau\left(\ell_{\mathrm{e}}+w_{0}-w\right) \cdot \exp \left(f \varphi_{\mathrm{c}}\right) \cdot\left(\cos \varphi_{\mathrm{c}}\right)^{s} \cdot H\left(\sigma_{\mathrm{u}}-\sigma_{\mathrm{f}, \max }\right),
$$

where

$$
\sigma_{\mathrm{f}, \max }=\frac{2 \pi r_{\mathrm{f}} \tau \ell_{\mathrm{e}}}{A_{\mathrm{f}}}
$$

The dashed curve in Fig. 4 demonstrates qualitatively how the fiber bridging function would look like in the case of finite fiber strength $\sigma_{\mathrm{u}}$ (the figure depicts a situation when the rupture of fiber is attained at force $P_{\mathrm{f}}=3.2 \mathrm{cN}$ ).

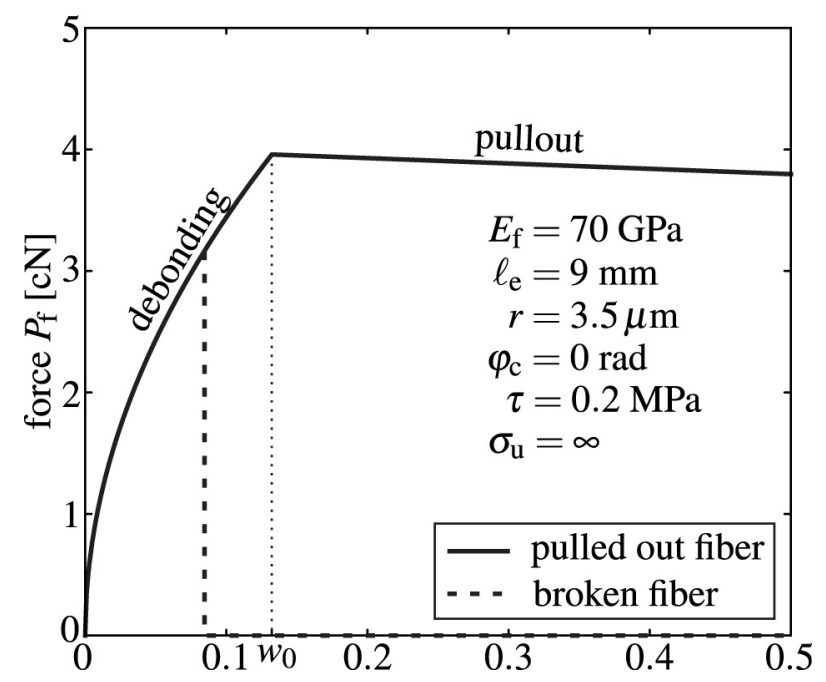

Fig. 4. Analytical model of a single fiber bridging action according to Naaman et al. [11] 
The complete filament bridging action (see Fig. 4) can be written as

$$
P_{\mathrm{f}}(w)=P_{\mathrm{f}, \mathrm{deb}}(w) \cdot H\left(w_{0}-w\right)+P_{\mathrm{f}, \text { pull }} \cdot H\left(w-w_{0}\right) .
$$

An example of the filament bridging action is depicted in Fig. 5a for material parameters that correspond to AR-glass fibers with random $\tau$ distributed uniformly between 0.01 and $0.4 \mathrm{MPa}$ and random fiber strength $\sigma_{\mathrm{u}}$ with Weibull distribution with shape parameter $m=5$ and scale parameter $s=1.75 \mathrm{GPa}$.
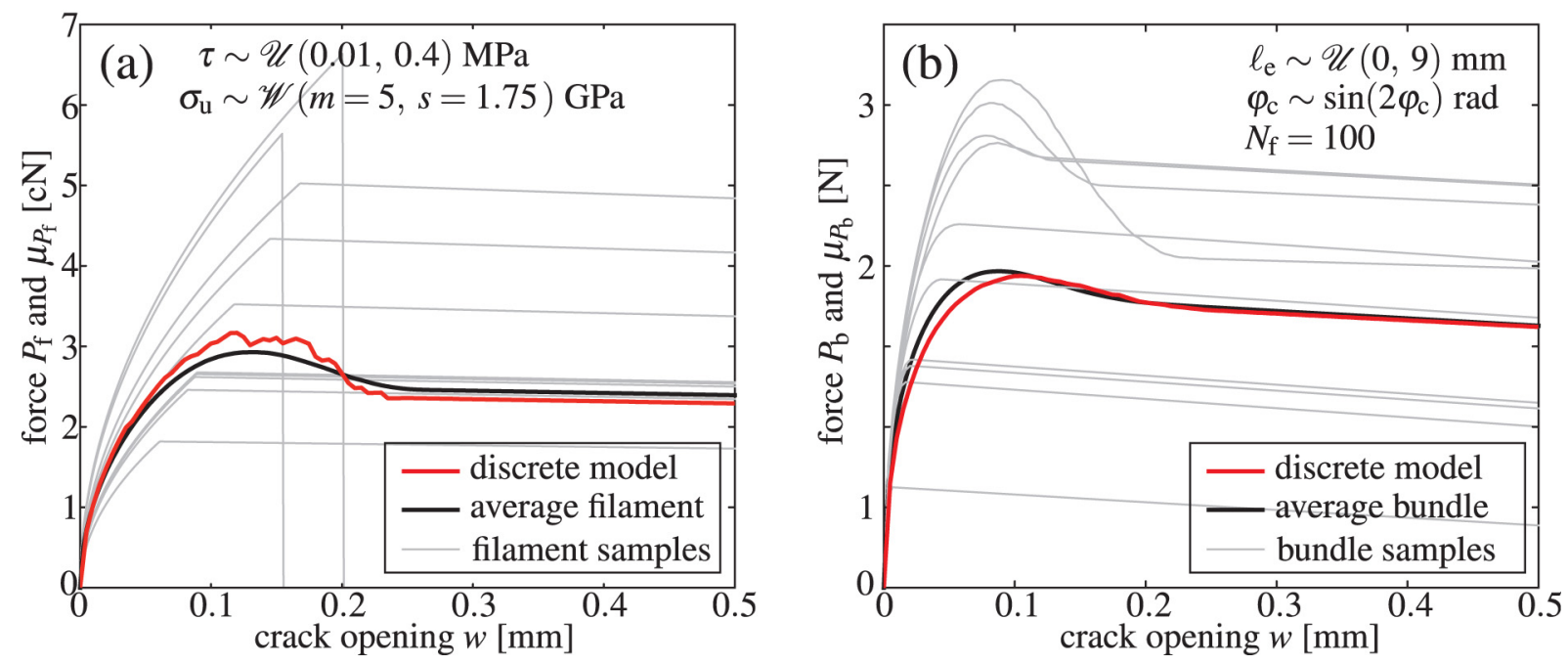

Fig. 5. Computational example performed with the present modeling framework: (a) single filament bridging responses (gray curves) sampled from the sampling space of random variables $(\tau \sim$ uniform distribution between 0.01 and $0.4 \mathrm{MPa}$ and $\sigma_{\mathrm{u}} \sim$ Weibull distribution with shape $m=5$ and scale $s=1.75 \mathrm{GPa}$ ) and the mean filament response (black curve); (b) filament bundle responses sampled from the sampling space of random variables $\left(\varphi_{\mathrm{c}} \sim \sin \left(2 \varphi_{\mathrm{c}}\right)\right.$ distribution and $\ell_{\mathrm{e}} \sim$ uniform distribution between 0 and $9 \mathrm{~mm}$ ) and the mean bundle response (black curve)

The filaments are embedded perpendicular to the crack plane in this example. The figure shows samples from the distributions given by (23) and the mean filament response given by (2), which, multiplied by the number of filaments in a bundle, is the prediction of the response of a perpendicularly embedded filament bundle. The red curve is a single simulation of a bundle consisting of 100 filaments performed by the discrete model.

Fig. $5 \mathrm{~b}$ depicts the bridging force of a bundle consisting of $N_{\mathrm{f}}=100$ filaments with random bond strength and fiber strength as in Fig. 5a but, additionally, the orientation angle and embedded length are considered as random variables. Random samples of such filament bundles and the mean bundle bridging force predicted by the probabilistic model with (7) are depicted. The red curve is the bridging force of $N_{\mathrm{b}}=100$ bundles that are randomly oriented and positioned within the crack predicted by the discrete model.

\section{Conclusions}

Both the probabilistic and the discrete model are capable of simulating the crack bridging action of chopped AR-glass strands in a cement-based matrix. The probabilistic model is computationally very efficient and able to evaluate statistical moments of the response. However, the model formulation includes a number of assumptions that make the model of use only for uniaxial tension in its current form. 
The discrete model evaluates the response of the composite as a single sample. Therefore, repeated calculations would have to be performed when the variability was of interest. The discrete model, even though more computationally demanding, is much more robust than the probabilistic model. It is not limited to uniaxial tension and is therefore suitable for general purposes. Its comparison with the probabilistic model serves as a verification of the semidiscrete fiber bundle implementation.

\section{Acknowledgements}

The financial support provided by the Ministry of Education, Youth and Sports of the Czech Republic under the project LO1408 'AdMaS UP - advanced Materials, Structures and Technologies' under 'National Sustainability Programme I' is gratefully acknowledged.

\section{References}

[1] Ball, H., 35 year review of the GRC technology, equipment and markets, In GRCA Congress, Istanbul, 2011.

[2] Biryukovich, K. L., Biryukovich, Y., Biryukovich, D. L., Glass fibre reinforced cement, CERTA Translation No. 12 Civil Engineering Research Assotiation, London, 1965.

[3] Bolander, J.E., Choi, S., Duddukuri, S.R., Fracture of fiber-reinforced cement composites: effects of fiber dispersion, International Journal of Fracture 154 (1-2) (2008) 73-86. https://doi.org/10.1007/s10704-008-9269-4

[4] Bolander, J.E., Sukumar, N., Irregular lattice model for quasistatic crack propagation, Physical Review B 71 (2005) 094106. https://doi.org/10.1103/PhysRevB.71.094106

[5] Kang, J., Kim, K., Lim, Y. M., Bolander, J. E., Modeling of fiber-reinforced cement composites: Discrete representation of fiber pullout, International Journal of Solids and Structures 51 (10) (2014) 1970-1979. https://doi.org/10.1016/j.ijsolstr.2014.02.006

[6] Kunieda, M., Ogura, H., Ueda, N., Nakamura, H., Tensile fracture process of strain hardening cementitious composites by means of three-dimensional meso-scale analysis, Cement and Concrete Composites 33 (9) (2011) 956-965.

[7] Lee, Y., Kang, S.T., Kim, J.K., Pullout behavior of inclined steel fiber in an ultra-high strength cementitious matrix, Construction and Building Materials 24 (2010) 2030-2041. https://doi.org/10.1016/j.conbuildmat.2010.03.009

[8] Li, V.C., Wang, Y., Backer, S., A micromechanical model of tension-softening and bridging toughening of short random fiber reinforced brittle matrix composites, Journal of the Mechanics and Physics of Solids 39 (5) (1991) 607-625. https://doi.org/10.1016/0022-5096(91)90043-N

[9] Majumdar, A. J., Ryder, J. F., Glass fibre reinforcement for cement products, Glass Technology 9 (1968) 78-84.

[10] Mobasher, B., Shah, S.P., Test parameters for evaluating toughness of glass fiber reinforced concrete panels, Materials Journal 86 (1989) 448-458.

[11] Naaman, A. E., Namur, G. G., Alwan, J. M., Najm, H. S., Fiber pullout and bond slip. I: Analytical study, Journal of Structural Engineering 117 (9) (1991) 2769-2790. https://doi.org/10.1061/(ASCE)0733-9445(1991)117:9(2769)

[12] Phoenix, S. L., Raj, R., Scalings in fracture probabilities for a brittle matrix fiber composite, Acta Metallurgica et Materialia 40 (11) (1992) 2813-2828. https://doi.org/10.1016/0956-7151(92)90447-M

[13] Roth, M. J., Eamon, C.D., Slawson, T.R., Tonyan, T. D., Dubey, A., Ultra-high-strength, glass fiber-reinforced concrete: Mechanical behavior and numerical modeling, ACI Materials Journal 2 (2010) 185-194. 
[14] Rypl, R., Chudoba, R., Scholzen, A., Vořechovský, M., Brittle matrix composites with heterogeneous reinforcement: Multi-scale model of a crack bridge with rigid matrix, Composites Science and Technology 89 (2019) 98-109.

https://doi.org/10.1016/j.compscitech.2013.09.014

[15] Schauffert, E., Cusatis, G., Lattice discrete particle model for fiber-reinforced concrete. I: Theory, Journal of Engineering Mechanics - ASCE 137 (7) (2012) 826-833. https://doi.org/10.1061/(ASCE)EM.1943-7889.0000387

[16] Vořechovský, M., Sadílek, V., Probabilistic determination of the number of fibers bridging a crack in short fiber reinforced composites, Bulletin of Applied Mechanics 7 (26) (2011) 21-25.

[17] Vořechovský, M., Sadílek, V., Rypl, R., Probabilistic evaluation of crack bridge performance in fiber reinforced composites, Engineering Mechanics 20 (1) (2013) 3-11.

[18] Wolf, W. W., Glass fiber reinforced cement, 1975. US Patent 3,902,912.

[19] Yip, M., Mohle, J., Bolander, J. E., Automated modeling of three-dimensional structural components using irregular lattices, Computer-Aided Civil and Infrastructure Engineering 20 (6) (2005) 393-407. https://doi.org/10.1111/j.1467-8667.2005.00407.x 